\title{
Myosin heavy chain, a novel allergen for fish allergy in patients with atopic dermatitis.
}

\author{
Yuka Shibata ${ }^{1}$, Serada Satoshi ${ }^{2}$, Taku Oishi $^{3}$, Kentaro Ohko $^{1}$, Mikiya Fujieda $^{3}$, Tetsuji Naka ${ }^{2}$, Shigetoshi Sano ${ }^{1}$ \\ 1. Dermatology, Kochi medical school, Nankoku, Kochi, Japan. \\ 2. The Center for Intractable Immune Disease, Kochi medical hospital, Nankoku, Japan. \\ 3. Pediatrics, Kochi medical school, Nankoku, Kochi, Japan.
}

Introduction

Fish allergy (FA) is the second most frequent type of food allergy in Japan. Fish allergens listed in the database of the WHO include parvalbumin, which is known to be the major allergen, $\beta$-enolase, aldolase $A$, vitellogenin and tropomyosin. Patients with atopic dermatitis (AD) have an increased risk for sensitization to allergens due to their increased skin barrier permeability. Here, we enrolled six patients with FA, who had also been diagnosed with $A D$ according to the diagnostic criteria of the Japanese guidelines.

\section{Method}

Fish protein extraction from salmon

$$
\downarrow
$$

$1 \mathrm{D}(\mathrm{A})$ and 2D(B) SDS-PAGE and Western blotting(WB)

$$
\downarrow
$$

Silver staining and In gel digestion

Mass spectrometric analysis (Nano LC-MS/MS)

Date analysis $\rightarrow$ detection of Myosin heavy chain(MYHC)

Preparation of recombinant salmon MYHC (rsMYHC)

WB(C), Inhibition assay(D), Basophil activation assay(E) with rsMYHC

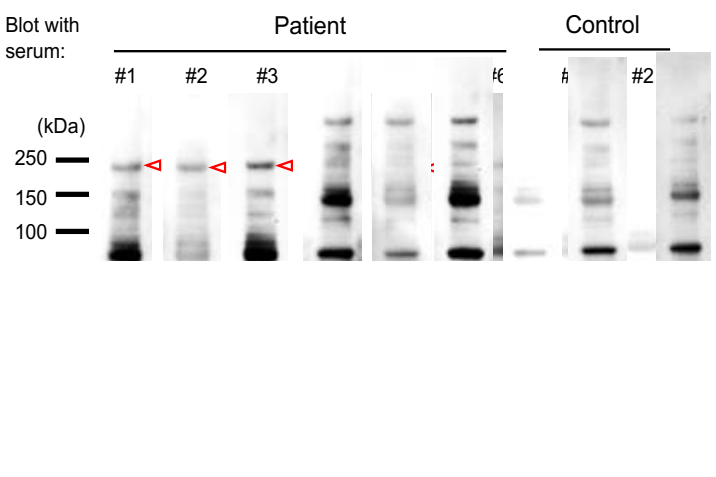

(A) Western blot using sera from FA patients and controls. Heat-untreated protein extracts from salmon were used for immunoblotting with sera from 6 patients and 2 controls, then were developed with anti-human IgE. Arrowheads, approximately $230-\mathrm{kD}$ proteins; \#, non-specific bands presumably derived from the second antibody, even in the absence of serum (data not shown).

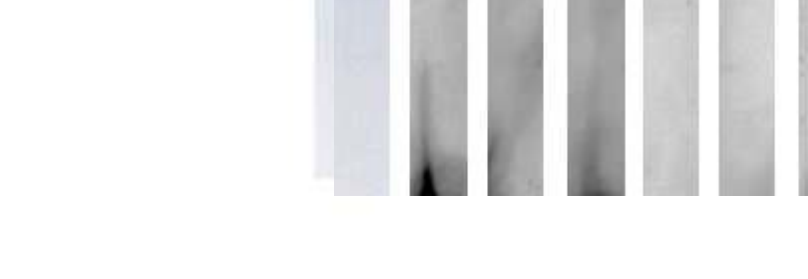

(C) Western blot using rsMYHC.

CBB, Coomassie Brilliant Blue R-250;

arrowheads, bands corresponding to rsMYHC.
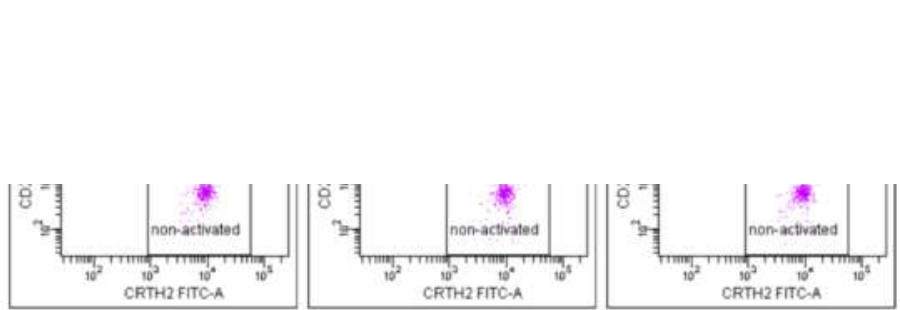

(E) Basophil activation test using rsMYHC

Peripheral blood from patient \#6 was used. Positive control, anti-lgE; negative control, PBS. rsMYHC, $10 \mu \mathrm{g} / \mathrm{mL}$. Numbers indicate the percentage of $\mathrm{CRTH} 2+\mathrm{CD} 203 \mathrm{c}+$ cells (arrows).

Clinical characteristics and serologic data.
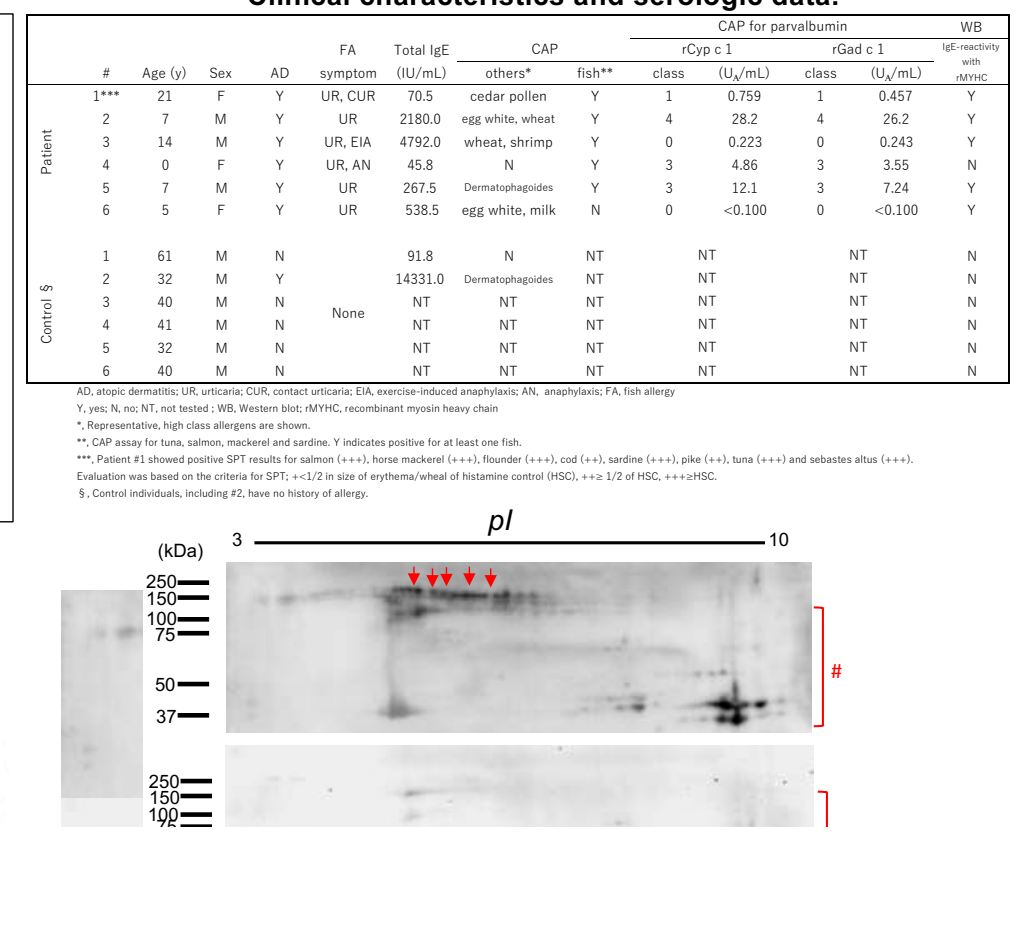

(B) Heat-untreated protein extracts separated by 2D-gel electrophoresis followed by IgE immunoblotting using sera from patient \#1 and control\#1. Five fractions of approximately $230-\mathrm{kD}$ proteins with different $p / s$ (red arrows) were isolated, then analyzed by nano LC-MS/MS and all were identified as MYHC. \#, aforementioned non-specific bands.

(1)

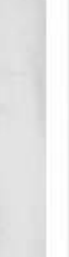

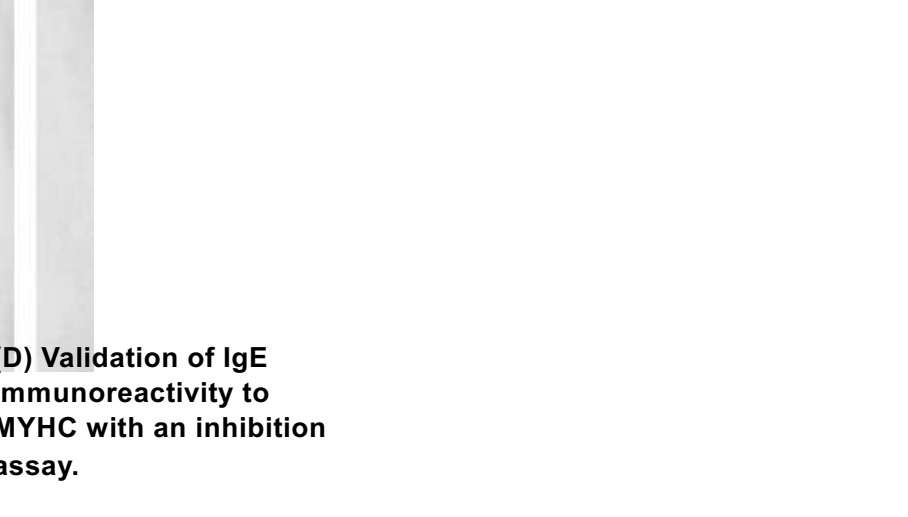

Sera from patients \#1 and \#3 were preincubated with or without the salmon protein extract, then Western blotted on rsMYHC. The specificity of MYHC was confirmed by restoration of the IgE reactivity when salmon protein extracts were used after absorption of MYHC with an anti-MYHC IgG antibody. Arrowheads, bands corresponding to rsMYHC; Asterisks, non-specific bands presumably derived from anti-MYHC IgG.

Conclusion
MYHC should be on the list of FA allergens to allow us to
make a correct diagnosis of FA, particularly in cases not
determined by conventional screenings with parvalbumin as
the representative allergen.
Raw fish cuisine has become very popular not only in Japan
but also outside of Japan. Touching raw fish might increase
the risk of sensitization to MYHC in patients with AD.

